Catalonian Health Institute (ICS) in an anonymized fashion from computerized data base. Demographic, clinical, functional, cervical intraepithelial neoplasia, and pharmacological variables were included. The primary outcome was PVV. A statistical analysis was carried out on the total population of women with active medical history in the territory between 01/01/2020-31/12/2020.

Result(s)* 23528 women were included with a mean age of $28.5 \pm$ ds7.7 years. The 35-40 years old group was significantly higher (29.1\%, p 0.001). 7.444 (31.6\%) women were registered as PVV. The average dose number was $1.76 \pm \mathrm{ds} 0.78$. The mean age of the vaccinated people was significantly lower than the unvaccinated $(20.0 \pm \mathrm{ds} 5.3$ vs $32 \pm \mathrm{ds} 5.9$, p <0.001). The PVV coverage showed significant differences by regions (30.5-41.1\%, p <0.001), health primary care teams $(28.2 \%$ $40.9 \%, \mathrm{p}<0.001$ ), and age groups: $85.7 \%$ in $15-19$ y-group vs $7.4 \%$ in 35-40y-group, p <0.001, inside Terres de l'Ebre. The 35-40 years-old cohort is the group with the lowest PVV coverage $(4.4 \%, \mathrm{p}<0.001)$

Disclosures The study protocol received ethics approval from the Ethical Committee Jordi Gol University Institute of Primary Care Research (Instituto Universitario de Investigación de Atención Primaria, IDIAP) code 21/064-P.

The data were obtained in an anonymized fashion provided by Information and Communication Technology Dept from the Minimum Basic Data Set at hospital discharge.

Conclusion* The study highlights women over 30 years-old as those at most risk because their low probability to PVV and/ or systematic HPV determination. Population dispersion and demographic structure may play a role as barrier in healthcare infrastructure and the implementation PVV.

\section{EFFICACY OF RRSO IN BRCA CARRIERS AND CLINICAL OUTCOMES OF FOLLOW-UP IN PATIENTS WITH ISOLATED STIC}

${ }^{1} \mathrm{G}$ Spagnol* ${ }^{*},{ }^{1} \mathrm{M}$ Noventa, ${ }^{1} \mathrm{G}$ Bonaldo, ${ }^{1} \mathrm{M}$ Marchetti, ${ }^{2} \mathrm{~S}$ Zovato, ${ }^{3} \mathrm{~L}$ Alessandrini, ${ }^{3} \mathrm{~A}$ Guerriero, ${ }^{2} S$ Tognazzo, ${ }^{1} \mathrm{R}$ Tozzi, ${ }^{1} \mathrm{C}$ Saccardi. ${ }^{1}$ Clinic of Gynecology and Obstetrics, University of Padua, Department of Women and Children's Health; ${ }^{2}$ Familial Cancer Clinic and Oncoendocrinology Veneto Institute of Oncology IOV - IRCSS, Padua; ${ }^{3}$ Surgical Pathology and Cytopathology Unit, University of Padua, Department of Medicine (DIMED)

\subsection{6/ijgc-2021-ESG0.543}

Introduction/Background* Serous Tubal Intraepithelial Carcinoma (STIC) is a non-invasive subtype of high-grade serous carcinoma (HGSC), usually located at the tubo-peritoneal junction and currently considered the precursor lesion of HGSC. The management of STIC diagnosed after Risk-Reducing Salpingo-Oophorectomy (RRSO) in women with BRCA-carriers remains unclear. The aim of our study was to evaluate the incidence of STIC, serous tubal intraepithelial lesions (STIL) and Occult-Cancer (OC) in patients submitted to RRSO and determine the long-term outcomes of these patients.

Methodology We conducted an observational retrospective study on patients with BRCA 1-2 mutation who undergone RRSO between January-2010 to Dicember-2020 at the Gynaecology Clinic of Padua. Inclusion criteria: (i) women with a negative pelvic examination prior to RRSO (ii) fallopian tubes analyzed using the Sectioning and Extensively Examining the Fimbriated (SEE-FIM) protocol. Exclusion criteria: patients with a positive gynecologic screening or with ovarian/tubal cancer prior to RRSO. We collected data about age,

\begin{tabular}{|c|c|c|c|}
\hline & $\begin{array}{l}\text { BRCA } 1 \text { carriers } \\
(n=80)\end{array}$ & $\begin{array}{l}\text { BRCA } 2 \text { carriers } \\
(n=73)\end{array}$ & $\begin{array}{l}\text { TOTAL } \\
(n=153)\end{array}$ \\
\hline Mean Age at RRSO* & $49.5 \pm 8.9$ & $51.8 \pm 9.3$ & $50.6 \pm 9.1$ \\
\hline Brest Cancer before RRSO* & $54(67.5 \%)$ & $49(67 \%)$ & $103(67 \%)$ \\
\hline Mean CA-125 & $13.8 \pm 6.8 \mathrm{IU} / \mathrm{mL}$ & $10.0 \pm 6.3 \mathrm{IU} / \mathrm{mL}$ & $12.3 \pm 6.7 \mathrm{IU} / \mathrm{mL}$ \\
\hline \multicolumn{4}{|l|}{ Menopausal Status } \\
\hline Pre-menopausal & $37(46 \%)$ & $19(26 \%)$ & $56(37 \%)$ \\
\hline Post-menopausal & $43(54 \%)$ & $54(74 \%)$ & $97(63 \%)$ \\
\hline \multicolumn{4}{|l|}{ Familiarity } \\
\hline Ovarian Cancer & $41(51.2 \%)$ & $19(26 \%)$ & $60(39.2 \%)$ \\
\hline Breast Cancer & $66(82.5 \%)$ & $51(70 \%)$ & $117(76.5 \%)$ \\
\hline Negative & $8(10 \%)$ & $7(9.6 \%)$ & $15(9.8 \%)$ \\
\hline
\end{tabular}

Legend: RRSO risk reducing salpingo-oophorectomy

menopausal status, history of breast carcinoma, pre-operative CA-125 levels, transvaginal-ultrasound features before surgery, and follow up (FUP) information after RRSO, specifically with CA-125 and gynecologic examination.

Result(s)* We included 153 patients: baseline characteristics (table 1). STICs was diagnosed in $4(2.6 \%)$ and STILs in 6 $(3.9 \%)$ patients. None patients with STIC and STIL underwent a restaging surgery or adjuvant chemotherapy; all patients were followed closely every 6 months with transvaginal-ultrasound and CA-125. None of them developed peritoneal carcinoma (PC) or primary peritoneal carcinomas (PPC) with a median FUP of 54.5 months (15-106) and 57,5 months (12-82) in patients with STIC and STIL, respectively. OC was diagnosed in 3 patients (2\%) and they underwent a staging-surgery; one patient developed a recurrence with PC after 18 months by staging surgery.

Conclusion* Considering the low incidence of OC-STIC-STIL, our data support the importance of RRSO in patients with BRCA 1-2 mutations for reducing the risk of ovarian cancer and for detecting the lesions in early stage. The management of patients with isolated STIC that is aimed to decreases the rates of subsequent PPC and PC. Our results demonstrated that a long-term close surveillance in patients with STIC should be considered a possible management strategy.

\section{LIBUSE TRIAL - ALGORITHM FOR CERVICAL CANCER SCREENING WITH USAGE OF HPV DNA TESTING WITH HPV 16/18 GENOTYPING AND P16/KI-67 DUAL-STAINED CYTOLOGY}

1J Sláma*, ${ }^{2} \mathrm{~V}$ Dvořák, ${ }^{3} \mathrm{M}$ Trnková, ${ }^{4} \mathrm{~A}$ Skrìvánek, ${ }^{5} \mathrm{~K}$ Hurdálková, ${ }^{5} \mathrm{M}$ Nováčková. ${ }^{7}$ First Faculty of Medicine, Charles University and General University Hospital, Department of Gynecology and Obstetrics, Prague, Czech Republic; ${ }^{2}$ Center of Outpatient Gynecology and Primary Care, Department of Outpatient Gynecology, Brno, Czech Republic; ${ }^{3}$ Aeskulab Pathology, Pathology, Prague, Czech Republic; ${ }^{4} G$-Centre, Department of Outpatient Gynecology, Olomouc, Czech Republic; ${ }^{5}$ Institute of Biostatistics, Masaryk University, Brno, Czech Republic

\subsection{6/ijgc-2021-ESGO.544}

Introduction/Background* The incidence and mortality of cervical cancer in the Czech Republic remains stable over more than 30 years irrespective of existing national screening based on annual collecting of Pap smears. The aim of our prospective trial was to evaluate the role of HPV DNA 
testing with 16/18 genotyping and triage with p16/Ki-67 immunocytochemistry.

Methodology Women between 30 and 60 years who had in 12 collaborating centres regular annual Pap smear were cotested in 3 years interval for HPV DNA with selective 16/18 genotyping (Cobas 4800, Roche). All HPV 16/18 positive cases and/or those with severe abnormality in cytology were directly refered to colposcopy; HPV non-16/18 positive cases and LSILs were triaged using p16/Ki-67 dual-stained cytology (CINtec Plus, Roche) and positive cases were refered to colposcopy.

Result(s)* Altogether 2407 patiens were eligible for analysis. Mean age of subjects was 43 years. The first round showed 8 cases with severe and 105 cases with mild Pap smear abnormalities. There were 7.4\% (180/2418) patients with HPV positivity, out of them 50 had HPV 16 and/or 18. Triage using p16/Ki-67 was positive in $22.5 \%$ cases (29/129). After 2 years of follow-up biopsy confirmed 38 HSILs and 2 glandular lesions, all of them were HPV positive.

Conclusion* Screening based on HPV testing with selective 16/18 genotyping and $16 / \mathrm{Ki}-67$ triage proved during three years four times more high-grade lesions including glandular lesions than standard screening based on Pap smears.

\section{PRIMARY PREVENTION OF OVARIAN CANCER: A PATIENTS DECISION AID FOR OPPORTUNISTIC SALPINGECTOMY}

${ }^{1} \mathrm{M}$ Gelderblom*, ' $\mathrm{L}$ Van Lieshout, ${ }^{1} \mathrm{~J}$ De Hullu, ${ }^{2} \mathrm{R}$ The, ${ }^{3} \mathrm{~A}$ Van Ginkel, ${ }^{4} \mathrm{~A}$ Oerlemans, ${ }^{5} \mathrm{~K}$ Smeets, ${ }^{6} \mathrm{M}$ Schreurs, ${ }^{7} \mathrm{~J}$ Piek, ${ }^{4} \mathrm{R}$ Hermens. ${ }^{1}$ Radboud University Medical Center, Department of Obstetrics and Gynecology, Nijmegen, Netherlands; 'ZorgkeuzeLab, Development and Implementation of Decision Aids, Delft, Netherlands; ${ }^{3}$ Rijnstate Hospital, Department of Obstetrics and Gynecology, Arnhem, Netherlands; ${ }^{4}$ Radboud University Medical Centre, Department of IQ healthcare, Nijmegen, Netherlands; ${ }^{5}$ Slingeland Hospital, Department of Obstetrics and Gynecology, Doetinchem, Netherlands; ${ }^{6}$ Medisch Spectrum Twente, Department of Obstetrics and Gynecology, Enschede, Netherlands; ${ }^{7}$ Catharina Hospital, and Catharina Cancer Institute, Department of Obstetrics and Gynecology, Eindhoven, Netherlands

\subsection{6/ijgc-2021-ESG0.545}

Introduction/Background* The discovery of the Fallopian tube epithelium as origin of high grade serous ovarian cancer has brought a new option for ovarian cancer prevention, the opportunistic salpingectomy (OS). The popularity of OS is increasing globally, however at present there is substantial practice variation. As a result, whether or not a woman is able to make her own decision on OS depends on the hospital or gynaecologist she visits. To lower practice variation, we developed and tested a patient decision aid (PtDA) for OS in women undergoing either pelvic gynaecological surgery with the intention to retain the ovaries or a sterilization.

Methodology We followed a systematic development process based on the International Patient Choice Aid Standard (IPDAS). Data were collected between June 2019 and June 2020, using both qualitative and quantitative methods. The development process took place in collaboration with patients and healthcare professionals, was overseen by a multidisciplinary steering group, and was divided in four phases; 1. Assessment of decisional needs using individual interviews and questionnaires; 2. Development of content and format based on decisional needs, current literature and guidelines; 3 . Alpha-testing and first revision round; and 4. Alpha-testing and second revision round.

Result(s)* An outline of the PtDA was developed based on decisional needs, current literature and guidelines. It became clear that the PtDA should consist of two separate paths: one on salpingectomy in addition to abdominal surgery and one on salpingectomy as a sterilization method. Both paths contain information on the anatomy and function of ovaries and Fallopian tubes, the estimated risk reduction of ovarian cancer and the potential benefits and risks of OS. Adjustments were made following alpha-testing round one. The improved PtDA was subjected to usability tests (alpha-testing round two), in which it scored an 'excellent' in patient testing and a 'good' in tests with gynaecologists.

Conclusion* In collaboration with patients and healthcare professionals, a PtDA was developed on OS. Both patients and gynaecologists thought it a usable aid which supports patients in making an informed decision whether to undergo an opportunistic salpingectomy, and supports the counselling process by gynaecologists.

\section{RISK REDUCTION SALPINGO-OOPHORECTOMY IN BRCA MUTATION CARRIERS. PRESURGICAL AND PATHOLOGY FINDINGS. A PROSPECTIVE COHORT STUDY}

${ }^{1} \mathrm{MDLR}$ Oliver*, ${ }^{2} \mathrm{~S}$ Aragon-Sanchez, ${ }^{3} \mathrm{~L}$ Alvaro, ${ }^{4} \mathrm{M}$ De Miguel-Reyes, ${ }^{5} \mathrm{E}$ Felipe-Pardo, 5J Montero, ${ }^{6} \mathrm{M}$ Martinez-Lopez, ${ }^{1} \mathrm{C}$ Álvarez-Conejo, ${ }^{1} \mathrm{G}$ López-Gonzalez, ${ }^{1} \mathrm{~B}$ Gil-lbañez, ${ }^{1} \mathrm{JM}$ Seoane-Ruiz, ${ }^{1}$ A Tejerizo-García. ${ }^{1}$ Hospital Universitario 12 de Octubre, Gynecology Oncology, Spain; ${ }^{2}$ Hospital Universitario 12 de Octubre, Breast Cancer, madrid, Spain; ${ }^{3}$ Hospital Universitario 12 de Octubre, Breast Cancer, Spain; ${ }^{4}$ Hospital Universitario 12 de Octubre, Genetics, Spain; ${ }^{5}$ Hospital Universitario 12 de Octubre, Spain; ${ }^{6}$ Hospital Universitario 12 de Octubre, Pathology, Spain

\subsection{6/ijgc-2021-ESG0.546}

Introduction/Background* Women with germline mutations in the BRCA $1 / 2$ genes have a lifetime increased risk of ovarian cancer, $36-63 \%$ and 10-27\% respectively. Accordingly, once childbearing is completed, Risk Reduction Salpingo-oophorectomy (RRSO) is recommended in this group of patients. The purpose of this study was to determine the presurgical findings and the incidence of Serous Tubal intraepithelial carcinoma (STIC) and occult carcinomas in BRCA mutations carriers in whom a RRSO was performed.

Methodology Prospective study that included patients with documented BRCA mutation who accepted RRSO between January 2011 to January 2021 at the Hospital Universitario 12 de Octubre. The study was approved by the ethics committee of the institution. During the month prior to surgery, a systematic ultrasound (US) and determination of serum $\mathrm{Ca}$ 125 levels were performed. Specialized gynecologists performed RRSO by laparoscopy. Unilateral or bilateral adnexectomy was performed according to the surgical history of each patient. Pelvic washing was done in all cases at the beginning of the procedure and tubes were removed at the uterine insertion. All the histologic exams were performed by pathologists subspecialized in Gynecologic Oncology and the sectioning and extensively examining of the fimbriated end protocol (SEE-FIM protocol) was applied. STIC was defined using a combination of morphologic evaluations to 